\title{
Itinéraires Itinéraires
}

Littérature, textes, cultures

2014-1 | 2015

Textualités numériques

\section{Écriveuses du Web : abondance et remix}

\section{Paule Mackrous}

\section{OpenEdition}

Journals

Édition électronique

URL : http://journals.openedition.org/itineraires/2276

DOI : 10.4000/itineraires.2276

ISSN : 2427-920X

Éditeur

Pléiade

\section{Référence électronique}

Paule Mackrous, «Écriveuses du Web : abondance et remix », Itinéraires [En ligne], 2014-1 | 2015, mis en ligne le 04 février 2015, consulté le 02 mai 2019. URL : http://journals.openedition.org/ itineraires/2276 ; DOI : 10.4000/itineraires.2276

Ce document a été généré automatiquement le 2 mai 2019.

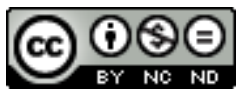

Itinéraires est mis à disposition selon les termes de la licence Creative Commons Attribution - Pas d'Utilisation Commerciale - Pas de Modification 4.0 International. 


\title{
Écriveuses du Web : abondance et remix
}

\author{
Paule Mackrous
}

\section{Introduction}

1 Dans un essai intitulé "The Wild, Wild Web: The Mythic American West and the Electronic Frontier» (2000), Helen McLure établit une relation entre l'imaginaire de l'Ouest américain et son cowboy, et une conception dualiste du Web. Dans l'Ouest, se côtoient le violent conquérant d'un territoire au nom d'intérêts économiques et individuels et l'aventurier libertaire qui découvre une aire de tous les possibles. Sur le Web, le scénario se traduit par deux manières d'agir bien différentes. D'un côté, il y a l'internaute qui cherche à tirer profit du réseau, de ses plateformes et d'un public consommateur. De l'autre, on retrouve celui qui, par des actes créateurs et aventuriers, inscrit ses actions dans une économie du don pour laquelle le public est considéré comme un créateur. Plus de dix ans après la publication de l'article de McLure, le « Wild Wild Web " (référant au Wild Wild West américain) et son cowboy demeurent des métaphores fortes pour analyser le Web. Mais qu'en est-il des cowgirls, dont on parle si peu? Qui sont-elles et quels sont leurs rôles sur le Web?

2 Cet article se penche précisément sur ces cowgirls, que nous appelons ici les écriveuses du Web (l'emploi du terme est justifié plus loin). Nous avons identifié deux notions permettant de problématiser leur pratique : l'abondance et le remix. Écrire sur le Web, c'est participer à l'abondance numérique, c'est-à-dire s'inscrire dans l'afflux de productions en ligne. Pour cela, les écriveuses trouvent des stratégies pour se connecter aux autres. Elles écrivent également pour garder le flux de leur créativité actif afin que leurs idées soient abondantes. Elles utilisent les différentes écritures médiatiques qui, parfois, engendrent des remix qui sont générés à partir d'une ou plusieurs autres productions culturelles. Ils peuvent aussi s'appliquer à l'identité des écriveuses, qu'elles remixent afin d'explorer des formes d'écriture singulières. 


\section{Les écriveuses du Web, les nouvelles cowgirls}

3 Dans son recueil de portraits de cowgirls, Teresa Jordan identifie plusieurs caractéristiques communes à ces figures oubliées de l'histoire américaine (1992). Les cowgirls sont des femmes indépendantes, confiantes et futées. Elles ressentent un attachement profond à la terre qu'elles s'approprient afin de se bâtir un avenir. Si elles n'ont pu compter sur l'histoire traditionnelle pour acquérir une visibilité, leur présence survit au sein des récits parallèles qu'elles ont elles-mêmes créés. Selon Jordan, elles sont des raconteuses d'histoires innées.

4 Pour cet article, nous avons réuni des femmes qui présentent les caractéristiques identifiées par Teresa Jordan. Ce sont des femmes qui s'approprient, avec confiance, indépendance et intelligence, les différentes plateformes du Web. Par là, elles se créent des espaces dans le réseau et, par ricochet, dans le monde. Puisque cet espace est toujours à défendre, à négocier et à réaffirmer, leur présence y est soutenue. L'expression écriveuses réfère ainsi directement à l'acte d'écrire: "qui sont en train d'écrire, là, maintenant ». Les écriveuses aiment écrire souvent et ne font pas nécessairement des œuvres, mais surtout des billets de blog, des tweets, des statuts Facebook. Elles s'intéressent profondément à leur terre, c'est-à-dire au Web lui-même, qu'elles connaissent en profondeur. Parfois écrivaines, artistes ou théoriciennes, ce sont avant tout, comme les cowgirls, des raconteuses d'histoires. Et ces histoires, elles les écrivent avec toute la panoplie d'écritures médiatiques.

\section{Abondance numérique : écrire pour se connecter}

5 L'écriveuse qui s'approprie un espace sur le Web fait face à un défi considérable : se créer un chemin dans l'abondance numérique. À l'heure actuelle, lorsqu'on explore le Web par le truchement d'un moteur de recherche, on se retrouve dans un espace virtuel qui ressemble étrangement au musée imaginaire d'André Malraux, c'est-à-dire un lieu dans lequel on observe "[...] une confrontation de métamorphoses qui se valent toutes " (1965 : 12). Le Web a exacerbé ce musée imaginaire qui se dote d'une quantité inouïe de sites, mais aussi d'images, de sons, de musiques, de vidéos et de textes de tous genres et tous rabattus sur un même plan et décontextualisés. La fameuse phrase: "À la représentation du monde, succède son annexion» (Malraux 1965: 72), pourrait être remplacée par : "À la représentation du monde, succède sa numérisation. » Alors qu'il saisit un mot-clé dans la fenêtre de son navigateur, l'internaute n'expérimente que la pointe de l'iceberg de cet afflux d'informations. À l'instant même où il explore les résultats de sa recherche, des sites web disparaissent tandis que d'autres sont créés.

Cela a des répercussions à plus petite échelle, évidemment. Dans la blogosphère, il y a plus de blogueurs que de lecteurs, remarque Kurt Lovink en citant Randi Mooney dans son ouvrage Zero Comments (2007 : 5). L'auteur révèle que les billets ne sont pratiquement pas lus (2007: 14). Quelques-uns sont relayés alors que les autres sont oubliés. Selon Olia Lialina, archéologue du Web et net artiste, c'est parce que les textes actuels sont très peu liés entre eux, contrairement à ceux du Web 1.0. Si le Web a survécu malgré les annonces récurrentes de sa mort prochaine, écrit-elle, ce qui est en voie de disparition, à l'heure du Web 2.0, c'est ce «fin tissage entre les productions en ligne ${ }^{1}$ ", c'est-à-dire l'hypertexte. Aujourd'hui, les « [...] internautes ne naviguent plus, ils cherchent dans Google » (Lialina 
2005). Cela effrite peu à peu cette expérience d'un Web où l'on naviguait par connexions. Les écriveuses $\mathrm{du}$ Web déjouent constamment la solitude liée à l'abondance numérique du Web 2.0. Cela, elles le font à la manière dont on utilisait le Web 1.0, en créant «[...] un réseau de connexions spontanées et de liens intimes » (Lialina 2005).

7 Penelope Trunk, blogueuse, romancière et femme d'affaires, estime que sur les blogs, tout repose dans la conversation et l'échange. Ses billets, qui reprennent les modalités de ses œuvres hypermédiatiques (qui ne sont plus en ligne) sont avant tout des récits de vie hypertextuels dans lesquels une vaste quantité de phrases et de mots sont liés à d'autres textes en ligne provenant d'autres blogs, sites ou articles. Dans sa pratique d'écriture, la frontière entre lire et écrire est pratiquement indiscernable : « le temps que je consacre à bloguer est en partie dédié à lire d'autres blogs et à créer des hyperliens vers ceux-ci » (Trunk 2007). Écrire sur le Web implique un dialogue et pour que celui-ci prenne forme, il faut lire les autres avec intérêt.

8 Selon Trunk, pour que le lien soit intime, il faut également se mettre à nu. Le contenu de son blog, qui accompagne la plateforme Brazen Careerist, ne porte pas uniquement sur les thèmes de la carrière et des affaires. Elle y dévoile sa vie quotidienne avec ses enfants et avec son mari surnommé the farmer. Les photographies insérées dans les billets de blog font voyager l'internaute de la cour arrière de la maison de l'écriveuse jusque dans sa chambre à coucher.

9 Le résultat est parfois troublant. En décembre 2011, elle publie une photographie dans laquelle on aperçoit le bas de son corps, dénudé, avec une ecchymose sur la hanche. Cette image accompagne un billet dans lequel elle raconte sa dispute avec le farmer: «Je suis dans un hôtel. Je pense que je vais mourir " (28 décembre 2011). Dans un billet intitulé «How to Know How Much to Reveal about Yourself? " (21 juillet 2009), Trunk expose des moments de son enfance qui est marquée par la violence, l'inceste et, surtout, par le silence. Atteinte du syndrome d'Asperger, dont l'un des principaux enjeux est la difficulté d'entrer en contact avec les autres, l'écriveuse justifie sa phobie du secret en affirmant 
qu'il a ruiné son enfance (Ibid.). Le dévoilement de soi est ainsi une nécessité que le Web lui permet de mettre en œuvre.

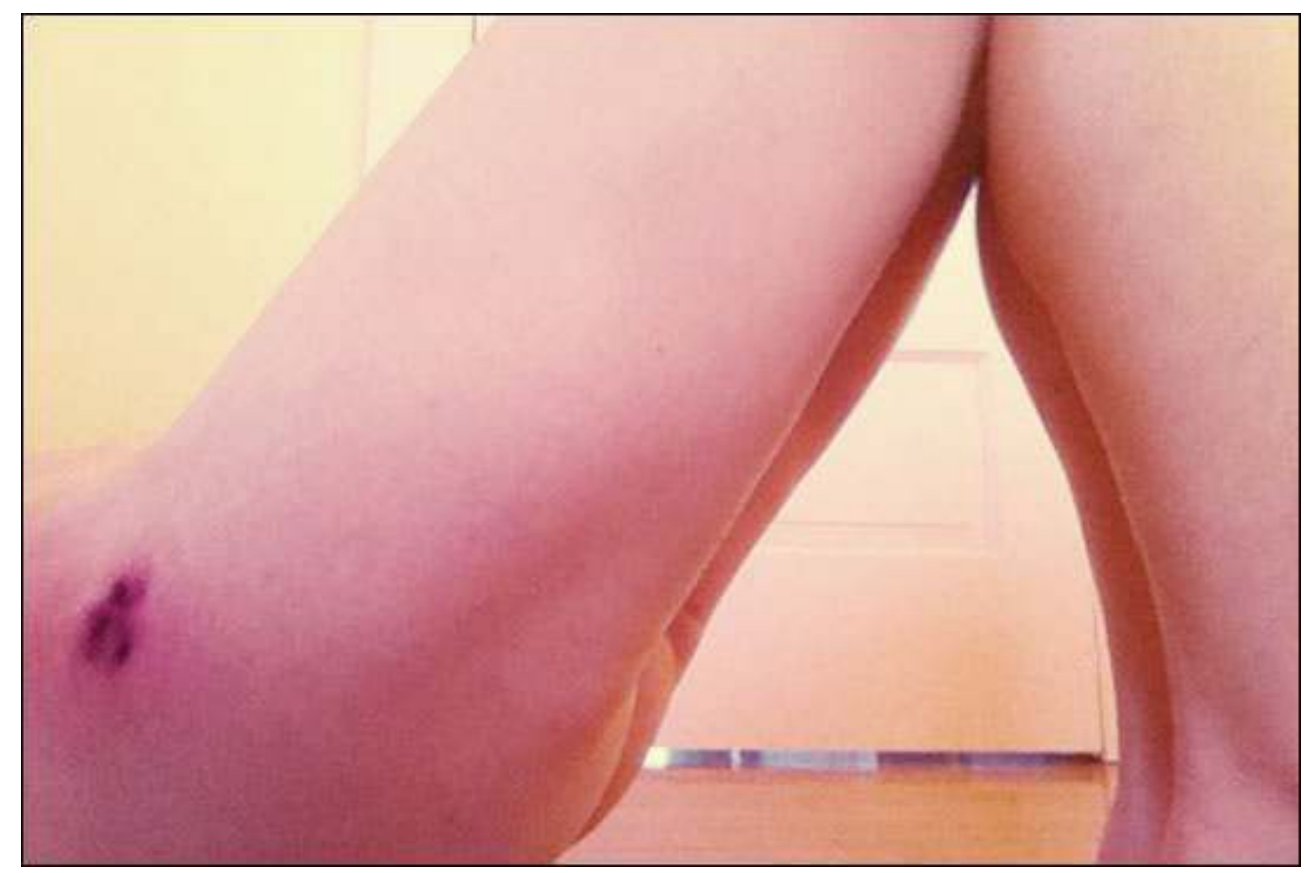

http://blog.penelopetrunk.com/2011/12/28/the-psychology-of-quitting/comment-page-2/

(c) Penelope Trunk

Ce déploiement de l'intimité sur le Web est souvent interprété non seulement comme du narcissisme (Lovink 2007 : 5), mais surtout comme un risque. Pourtant, tel que l'affirme la musicienne et blogueuse Amanda Palmer, il s'agit surtout d'un acte de confiance. Ce n'est que par cet abandon de soi que l'écriture peut devenir un lieu de connexions. Pour ses interventions sur les réseaux sociaux et sur son blog, Amanda Palmer s'inspire de son expérience acquise dans les rues de la ville de New York. Selon elle, la grande ville ressemble en tout point au Web et à son abondance. Dans une vidéo intitulée The Art of Asking (2013b), Palmer raconte l'une de ses premières expériences de travail alors qu'elle se déguisait en statue de mariée (Eight Foot Bride), toute peinte de blanc et fleur à la main. Son travail consistait à créer une connexion visuelle avec les passants dans la rue et à leur tendre une fleur. En échange de cette offrande en apparence éphémère, certains passants lui donnaient de l'argent. L'écriture sur son blog et sur Twitter lui permet de générer ce même type de proximité aléatoire avec les autres :

Ma carrière en musique est toute liée à mes efforts pour rencontrer des gens sur Internet [...] en bloguant, en tweetant, pas uniquement au sujet de mes tournées ou de mes vidéos, mais à propos de mon travail, de mon art, de mes peurs, de mes erreurs. (Ibid.)

L'écriveuse affirme : «Lorsque nous regardons l'histoire, nous constatons que les artistes ont toujours fait partie de la communauté [...], avant aujourd'hui, ils n'étaient pas des étoiles intouchables» (Ibid.). La confiance aux autres, selon Palmer, est le ciment des communautés. Les communautés permettent à l'écriveuse, à ses textes et à sa musique de survivre dans l'abondance numérique. Ce qui importe, pour Palmer, est que «[...] nous nous voyions vraiment les uns les autres", car "lorsque nous nous voyons vraiment, nous voulons nous entraider » (Ibid.). Les plateformes en ligne lui permettent d'exercer son travail d'artiste en développant une forte communauté d'admirateurs. Ainsi, Palmer 
va jusqu'à pratiquer le couchsurfing chez des admirateurs rencontrés sur Twitter. Offrant sa musique et ses textes gratuitement sur le Web, de la même manière dont elle tendait autrefois une fleur aux passants, elle invite les gens à lui verser de l'argent. Elle considère alors les dons et les invitations comme des actes de réciprocité volontaire.

Chez Trunk comme chez Palmer, écrire dans l'abondance numérique engendre une abondance de connexions avec les autres. Les dons, autant du côté des écriveuses que des internautes, sont avant tout des «[...] dispositifs servant à construire des connexions avec les autres. Ils créent des relations, et se fondent sur ces relations » (Lessig $2008: 148)$. Audelà d'une stratégie qui est largement récupérée par le marketing, le don devient, pour Palmer comme pour Trunk, un véritable mode de vie. Dans leur cas, il s'agit également d'un mode de survie à différents niveaux : financier, social, psychologique.

\section{Flux : écrire pour l'abondance}

13 Chez les écriveuses, écrire dans l'abondance numérique c'est aussi écrire pour l'abondance: de points de vue, d'idées, de revendications. L'écriture sur le Web est souvent décrite en fonction d'un sentiment de liberté lié à une absence de médiation, et cela depuis le tout début de son existence. En 1998, alors que les ordinateurs personnels commencent réellement à proliférer dans les foyers, Aliza Sherman alias Cybergrrl, écrit à propos de sa découverte du Web: "Je n'avais pas à demander à quiconque une permission ni à suivre aucune règle afin de publier. C'était une idée envoûtante » (1997 : 8). Les blogs sont des lieux de liberté où « les femmes peuvent écrire contre la censure et contre le fait d'être ignorées ou ostracisées ", estime la blogueuse et essayiste Kate Zambreno (2007 : 291). Les écriveuses officient non seulement sur les blogs, mais aussi sur les réseaux sociaux, sur les forums ou dans des courriels : « [...] on utilise tous les canaux possibles par lesquels on peut crier, chanter, s'enflammer » (Zambreno $2007: 296)$. Écrire sur le Web, c'est l'opportunité, mais non l'obligation, de dévoiler ce qu'il y a d'indomptable en soi-même. Cela permet de ne pas se sentir bloquée. L'abondance numérique suscite l'abondance dans le flux créatif et vice versa.

Chez les écriveuses, l'abondance n'est pas perçue comme une accumulation, mais comme une continuité, un flux nécessaire à la pensée. Julia Cameron, spécialiste des blocages créatifs, suggère à tous d'écrire au moins trois pages tous les matins. Elle appelle cette pratique «les pages du matin» (2007: 25). Le principe est le suivant: «[...] écrire strictement le flux qui traverse la conscience » (Ibid.). De manière similaire, sur le Web, il n'est pas rare que l'on privilégie la quantité plutôt que la qualité. Sur un blog accompagnant la rédaction de sa thèse, la doctorante Lisa Smithies se fixe des objectifs qui n'ont rien à voir avec la qualité d'une proposition théorique : «Mon but est d'écrire un billet au moins une fois par semaine. Avertissement : ce blog peut contenir des folies et des jurons à l'occasion " (janvier 2013). Sur ce blog, on rencontre des digressions qu'on ne retrouvera jamais au sein d'une thèse ou d'un article scientifique. Celles-ci sont pourtant nécessaires dans le processus qui mène à l'innovation.

Les discussions en ligne jouent également un rôle important dans la compréhension de la notion d'abondance. Ne sommes-nous pas toujours en train d'écrire, sur le Web? Les forums, les chats ou les réseaux sociaux permettent d'aller trouver rapidement de l'information sur un sujet qui requiert un soutien technique ou émotionnel. On y partage et on y discute de nos expériences personnelles ou de ce que nous sommes en train de 
faire. Ce sont des espaces de déblocages, de confidences et de réconfort. Ce sont des lieux de connexions ou encore, de distractions. $\mathrm{Si}$, lors de la rédaction d'un texte, un problème technique ou un blocage créatif se présente, il est toujours possible d'aller écrire ailleurs. Nous n'avons pas à être seules dans un processus de rédaction. Cette écriture de l'échange devient le moteur d'une écriture en continu. Tel que l'écrit Geneviève Pettersen alias Madame Chose, blogueuse et romancière: "Quand je travaille beaucoup je passe beaucoup de temps sur Facebook. C'est ma façon de ventiler et de prendre des pauses entre les phrases " (septembre 2013). Si cette forme d'errance est parfois dévoreuse de temps, elle se présente aussi comme une stratégie permettant de retourner avec plus d'aisance à la rédaction.

N'oublions pas que les textes que nous publions sur le Web, aussi courts soient-ils, font immédiatement partie du musée imaginaire du Web. On ne peut aborder cette abondance sans s'attarder à l'ambivalence que génère le fait de rendre publics des réflexions et des échanges spontanés. Cela engendre un mélange d'excitation et de saturation, de nécessité et de superflu, de curiosité et de dépendance. On retrouve cette ambivalence de manière récurrente dans les écrits de Kate Zambreno où la liberté prometteuse du Web trouve des limites dans la fidélité d'un lectorat. Les attentes des lecteurs s'inscrivent alors dans le processus d'écriture. Zambreno veut continuer d'être lue, appréciée et commentée: "Maintenant, raconte l'écriveuse, je suis beaucoup plus consciente de mon lectorat et lorsque personne ne commente, je me demande si c'est parce que j'ai transgressé des limites en écrivant à propos de X, Y, Z» (Zambreno 2007 : 290). On peut alors effacer et éditer son texte ad infinitum.

Les écriveuses se servent parfois des réactions des internautes comme moteur pour l'écriture. À propos d'un personnage inventé sur son blog, Geneviève Pettersen écrit que ce sont les lecteurs «[...] qui voulaient en savoir plus sur elle, sur François et sur les autres: alors je continue cette histoire pour eux [...] Même quand je n'ai pas envie d'écrire, ce sont les lecteurs qui m'y poussent » (2013:31).

Puisque les écriveuses publient leurs textes dans l'abondance numérique, il arrive que leurs lecteurs soient subitement abondants. Une soudaine popularité offre un autre regard sur un texte rédigé spontanément. Au sujet d'un de ses billets de blog, Palmer écrit : « Si j'avais su que 250000 personnes allaient lire ce billet, j'aurais écrit quelque chose de vraiment meilleur, mais si j'avais écrit quelque chose de vraiment meilleur, je ne l'aurais jamais écrit » (2013a). Les meilleures idées émergent parfois au beau milieu d'une panoplie de réflexions spontanées, maladroites ou mal formulées.

Lorsque les écriveuses écrivent pour l'abondance, elles luttent contre l'idée d'une réflexion arrêtée. C'est ce qui permet, en retour, à la pensée de ne pas se figer, de continuer à se développer. Par là, les écriveuses créent un Web qui ne sera jamais un territoire conquis, mais toujours une source d'explorations créatives. Elles animent des espaces qui évoluent à la fois par et pour l'écriture. Écrire pour l'abondance, c'est un acte d'écriture en continu qui vise résolument à ne pas tourner le dos à l'enthousiasme et à la multitude de points de vue.

\section{Remix : écrire pour décoder}

L'abondance numérique a aussi fait naître ce que Lawrence Lessig appelle une culture remix (Lessig 2008). De manière générale, le remix consiste à combiner ou à éditer $\mathrm{du}$ 
matériau préexistant pour créer quelque chose de nouveau (Navas 2010). La notion est empruntée à l'univers de la musique, plus précisément à la pratique du Disc Jockey et des musiciens de hip-hop qui réutilisent des portions de pièces musicales pour créer une composition originale. On peut lier plus généralement le remix à ce qu'on appelle, depuis au moins la fin des années 1960, la citation dans les arts visuels. Comme celle-ci, le remix «[...] interroge les différentes modalités selon lesquelles les œuvres réinvestissent et réfléchissent celles qui les ont précédées » (Beylot $2004: 25$ ).

21 Le numérique permet plus que jamais à tous de s'approprier des éléments de sa culture pour les transformer, les interpréter, les partager. On peut littéralement écrire avec les images, les sons, les vidéos, plutôt que simplement écrire à propos de ceux-ci : ce qui requiert une connaissance tout aussi approfondie de ces mêmes éléments. Outre les productions publiées sous licence Creative Commons ou Copy Left qui rendent les processus d'appropriation d'images, de vidéos, de textes ou encore de sons, légaux par leur auteur, beaucoup d'éléments de culture en ligne sont récupérables par un seul clic de souris. Les écriveuses remixent constamment leur culture.

Elisa Kreisinger, hacker et cyberféministe, remixe des productions de la culture populaire malgré leur protection par le droit d'auteur. Elle transforme les hommes de la série Mad Men en féministes ou encore les femmes de l'émission de téléréalité Real House Wives en lesbiennes. L'éthique du hacker, tout comme celle des écriveuses, « revendique la liberté personnelle de comprendre comment les choses fonctionnent en soulignant des failles dans le système duquel nous dépendons tous » (http://www.popculturepirate.com/). En utilisant les éléments de culture et en les critiquant de l'intérieur, Kreisinger décode et déconstruit les idéologies qu'ils renferment. Partageant par la suite ses remix, elle devient une actrice dans sa culture plutôt qu'une simple consommatrice ou commentatrice. Dans un billet intitulé « Why Women Make the Best Remixers » (2011), Kreisinger écrit que les femmes sont généralement peu représentées dans l'histoire culturelle. Cela est le résultat direct des idéologies patriarcales qui ont mené le monde et la culture jusqu'aujourd'hui. Elle affirme ainsi que le «[...] remix est une stratégie légale et juste pour transformer le contenu créé par les patriarches afin d'inverser leurs stratégies » (cité dans Coppa 2010).

Une action remix ne résulte pas nécessairement d'un acte de piratage. Elle peut se concrétiser dans le fait de relayer et de rendre hommage à quelque chose, pour contribuer à sa visibilité dans l'abondance numérique. Kevin Kelly est le premier à avoir mentionné le terme movage, pour parler de l'archivage de sites web. Dans un cours billet, il affirme que : «La seule manière d'archiver de l'information numérique est de la faire voyager plutôt que de la stocker " (2008). L'idée de movage a une portée plus large que le simple fait de transférer du contenu sur de nouvelles plateformes. Elle part du principe que, plus des productions culturelles sont remixées, plus elles survivent longtemps. Pour survivre, les éléments de culture doivent faire l'objet d'une interprétation toujours renouvelée. S'ils s'inscrivent dans la durée, ils ont plus de chance d'entrer dans l'histoire.

Dans un Tumblr intitulé Women as Objects, Kate Durbin s'approprie et rassemble des vidéos et des notes créées par des adolescentes sur les plateformes Tumblr. Le fait de rassembler autour d'un thème commun et sur une même plateforme des éléments de culture engendre une archive. Celle-ci permet de mettre en valeur l'esthétique propre au langage visuel et textuel des adolescentes : «[...] le résultat est un portrait étonnamment honnête de ce que cela signifie d'être une ado " (Jones 2012), peut-on lire au sujet de son projet. Selon l'auteure, ces adolescentes sont «[...] vraiment inspirantes, talentueuses et 
tellement ouvertes à partager leur vie " (Ibid.). Le remix permet littéralement de leur livrer un hommage perpétuel, jour après jour.

Plutôt que de simplement écrire un texte au sujet de cette archive, le Tumblr de Durbin se décline à son tour en d'autres formes de remix: une performance, deux œuvres vidéographiques et une conférence. Tout comme les remix de Kreisinger, ceux de Durbin incarnent les principes du media litteracy, un ensemble de compétences permettant entre autres d'analyser et de créer à travers une panoplie d'écritures médiatiques. Bien plus qu'un simple divertissement, la pratique du remix est, pour les "écriveuses», une stratégie politique. Elle permet «[...] la récupération des images oppressives pour créer une vision positive d'une société juste» (Coppa 2010) et/ou elle forme une archive vivante de ce que l'on souhaite garder.

\section{Remix identitaire : écrire pour éprouver}

Comme la culture, l'identité est aussi quelque chose que l'on peut s'approprier et modifier pour former un remix identitaire. Selon Paul D. Miller : «L'identité réfère à l'idée de se créer un environnement dans lequel le monde agit comme s'il était notre propre réflexion" (2006: 61). À la lumière de cette définition, nous articulons le remix identitaire à travers trois principes importants. Premièrement, chaque création identitaire engendre une interprétation singulière du monde et permet ainsi d'explorer en profondeur un ou plusieurs aspects de son propre rapport au monde. Deuxièmement, cette création est infinie, car, sur le Web et dans l'interaction, le personnage devient une source d'actions imprévues pour celle qui l'incarne. Troisièmement, et c'est là un point fondamental, le remix identitaire implique que l'identité est malléable. Il devient un moyen par lequel on lutte contre la rigidité causée par une identité trop définie. Il permet d'«éprouver " dans les deux sens du terme, c'est-à-dire de mettre à l'épreuve de nouvelles formes d'écriture et de ressentir, par l'écriture, des émotions négatives comme positives trop souvent anesthésiées par le quotidien et les normes sociales.

L'écriveuse derrière le personnage web Victoria Welby offre une seconde vie à une auteure oubliée de la fin du xIx ${ }^{\mathrm{e}}$ siècle. Sur le Web, la sémioticienne devient une féministe partageant, entre autres, des réflexions féministes de son cru ou non, des récits érotiques ou encore ce qu'elle appelle des dérives. Bien qu'on reconnaisse sa posture singulière et engagée, Victoria Welby demeure un personnage « [...] en constante construction et qu'on ne peut définir qu'en admettant la logique du tiers inclus " (Welby, site Web). Se déclinant en une multitude de projets en ligne et infiltrant plusieurs plateformes, Welby crée librement et, par là, éprouve librement : du plaisir intense, de la colère lucide ou encore de la douleur profonde.

Sur un de ses blogs intitulé Les identitaires, blog qu'elle définit comme "un ensemble de tableaux relevant ou l'absurdité du monde ou sa beauté improbable ", Welby décode des situations de la vie en apparence banales. Par ces courts récits, elle met en évidence les petites violences quotidiennes que nous ne voyons plus tant elles sont intégrées dans les habitudes et les mœurs. En écrivant à propos d'un homme qui l'accoste dans la rue, elle écrit: "Je me demande ce que je trouve le plus violent: son entêtement à lui à m'interpeller ou le fait que son comportement est trop souvent considéré comme bénin, anodin » (août 2013). Par ce genre de réflexions, elle donne aux lectrices l'occasion de ressentir ce qui, dans le quotidien, se dissimule sournoisement, mais fait des ravages. 
Qu'elles remixent des productions culturelles ou leur propre identité, les écriveuses opèrent toujours une forme de décodage. Celui-ci apparaît dans les écrits d'Albertine Bouquet comme une lutte contre l'éblouissement. Personnage d'un projet littéraire en ligne mené par les blogueuses Julie Boulanger et Amélie Paquet, Albertine Bouquet écrit : «[...] je lis des livres et des blogues parce que je veux connaître l'être humain qui écrit et qui a des choses à dire. Je ne veux pas être éblouie par des concepts de merde " (septembre 2010). Décriant la rigidité dans laquelle se limitent les auteurs de «blogues à concept ", Albertine déplore également l'attitude convenue et autoritaire qu'adopte une partie du milieu littéraire québécois. bande dessinée, le personnage raconte des événements de sa vie à travers lesquels sont dénoncés des violences, des injustices et, surtout, des aveuglements. Rédigés dans un excès émotif, du moins, c'est ce que le lecteur s'imagine, ses billets transmettent des analyses tranchantes et passionnées du milieu des arts, de la littérature ou encore du milieu universitaire. Le personnage écrit avec démesure et cette démesure peut parfois être interprétée comme de la folie. C'est là que réside toute la force de ses réflexions. Albertine préfère la passion à la modération, la singularité à l'uniformisation, l'émotivité à la rationalisation. Mais cela, elle ne fait pas que l'écrire, elle l'incarne. Par la fougue qui anime ses textes, Albertine nous rappelle sans cesse que l'une des plus grandes violences se trouve dans le fait de ne pas pouvoir s'exprimer librement, ce qui revient trop souvent à ne pas pouvoir éprouver librement.

31 La rigidité de l'identité peut aussi être combattue par l'autodérision. Le personnage Twitter de Melissa Broder, poète américaine, publie des tweets dans lesquels il met souvent en scène sa propre relation aux technologies numériques. On y trouve de nombreux petits vers autoréflexifs qui soulignent la vacuité de notre rapport au Web tels que : «Nous sommes désolés, mais votre mot de passe doit contenir la sensation d'être une personne entière ", remixant les formulaires de mots de passe en ligne. Un autre jour, elle écrit : «Un amour comme dans 0 notification », référant aux notifications Facebook que nous attendons parfois vainement après la publication d'un statut. Les petits vers de Broder, comme l'affirme si justement Ellen Frances, causent «[...] un TKO (Knockout technique) avec quelques mots à chaque tweet, sans exception " (janvier 2013). Bien différente de celle que l'on retrouve dans ses recueils, la poésie Twitter de Melissa Broder s'inscrit parmi une panoplie d'autres tweets à travers lesquels ils font saillie, créant une résonance directe avec l'expérience hic et nunc de l'internaute.

Ouvrant une fenêtre sur un aspect de leur personnalité et l'exagérant par la consolidation d'un personnage, les écriveuses du Web touchent quelque chose d'universel. Ce quelque chose ne parle plus d'elles-mêmes, mais bien souvent d'une émotion qui se dissimule derrière une identité rigide, une personnalité anesthésiée. Cela, elles le font en se regardant attentivement. Il ne s'agit pas de narcissisme. Le narcissique, par une attention soutenue, s'admire. Bien au contraire, les écriveuses dévoilent également ce que Zambreno appelle le "Ugly Self» (le soi laid) par lequel elles se donnent le droit d'éprouver leur écriture comme leurs émotions et, par là, d'ouvrir cette possibilité pour les autres. 


\section{Conclusion : écrire pour faire suivre}

33 malléable, le cyberespace est créé différemment par toutes celles et tous ceux qui
pensent, parlent et, écrivent à son sujet » $(2000: 459)$. Nous ajouterions qu'il est surtout créé par celles qui le connaissent de l'intérieur. Les écriveuses créent avec les modalités et les spécificités du Web que sont l'abondance numérique, le flux et le remix culturel ou identitaire. Par là, elles peuvent se connecter aux autres, encourager la multitude de points de vue, poser des actions au sein de leur culture, se donner la chance d'éprouver des émotions intenses et de mettre en œuvre des formes d'écriture différentes. Elles maintiennent une présence continue dans l'abondance numérique en s'appropriant des espaces qui demeurent publics, visibles pour tous, en tout temps.

Les histoires des écriveuses, peu importe leur forme, appellent le " faire suivre », un geste par lequel ces histoires prolongent leur durée de vie, parce qu'il est lui-même un acte d'écriture. Alors que nous retweetons spontanément les vers de Melissa Broder, que nous likons un dessin d'Albertine sur Facebook, que nous ajoutons une vidéo d'Elisa Kreizinger à notre liste de lecture sur YouTube ou que nous envoyons un billet de blog de Zambreno par courriel, nous sommes en train d'écrire avec elles. Nous sommes également en train de nous inscrire, par elles, sur le Web. Les écriveuses tracent ainsi des chemins singuliers dans un Web d'autopromoteurs, de plateformes formatées et de capitalisme exacerbé.

\section{BIBLIOGRAPHIE}

Beylot, Pierre, 2004, Emprunts et citations dans le champ artistique, Paris, L'Harmattan.

Bouquet, Albertine, 2010, « La blogueuse ", Albertine retrouvée. Récits d'une catin cérébrale, http:// desir-obscur.livejournal.com/143823.html, consulté le 9 janvier 2015.

Broder, Melissa, 2012, Melissa Broder, [Blog], http://www.melissabroder.com/page/3/, consulté le 9 janvier 2015.

Broder, Melissa, 2012, Meat Heart, Baltimore, Publishing Genious Press.

Cameron, Julia, 2007, The Complete Artist's Way. Creativity as a Spiritual Practice, New York, Penguin.

Coppa, Francesca, 2010, « Interview with Elisa Kreisinger », Transformative Works and Cultures, $\mathrm{n}^{\circ}$ 5, [En ligne], DOI : http://dx.doi.org/10.3983/twc.2010.0234, consulté le 9 janvier 2015.

Frances, Ellen, 2013, « Melissa Broder IRL on Video Art, Poetry, Gifs \& Soft Grunge », Everyday Genius, [Blog], http://www.everyday-genius.com/2013/01/ive-been-super-obsessed-withmelissa.html, consulté le 9 janvier 2015.

Hybenova, Katerina, 2012, « Melissa Broder: Twitter is Another Artform », Brooklyn 365, [Blog], http://brooklyn365.com/2011/01/melissa-broder-twitter-art-form/, consulté le 9 janvier 2015.

Itinéraires, 2014-1 | 2015 
Jones, Daniel, 2012, « Women As Objects: The Tumblr Teen », Electronic Beats, [Blog], http:// www.electronicbeats.net/en/features/women-as-objects-the-tumblr-teen/, consulté le 9 janvier 2015.

Jordan, Teresa, 1992, Cowgirls. Women of the American West, New York, Bison Book.

Kelly, Kevin, 2008, « Movage », The Technium, http://kk.org/thetechnium/archives/2008/12/ movage.php, consulté le 9 janvier 2015.

Kreisinger, Elisa, 2011, « Why Women Make the Best Remixers », [Blog], http://

elisakreisinger.wordpress.com/2011/10/14/3-reasons-why-women-make-the-best-remixes/, consulté le 9 janvier 2015.

Lessig, Lawrence, 2008, Remix: Making Art and Commerce Thrive in the Hybrid Economy, Londres, Bloomsbury Academic.

Lialina, Olia, 2005, «A Vernacular Web. The Indigenous and the Barbarians », [Site web], http:// art.teleportacia.org/observation/vernacular/, consulté le 9 janvier 2015.

-, 2007, « Vernacular Web 2 », Contemporary Home Computing, [Site web], http://contemporaryhome-computing.org/vernacular-web-2/, consulté le 9 janvier 2015.

-, 2010, « Vernacular Web 3. Prof. Dr. Style », Contemporary Home Computing [Site web], http:// contemporary-home-computing.org/prof-dr-style/, consulté le 9 janvier 2015.

Lovink, Geert, 2007, Zero Comments: Blogging and Critical Internet Culture, New York, Routledge.

Mackrous, Paule, 2007, « Le partage sur le Web : personnages virtuels et Web 2.0 selon Martine Neddam », .dpi, $\mathrm{n}^{\circ}$ 15, [En ligne], http://dpi.studioxx.org/demo/?q=fr/no/15/partage-Webpersonnages-virtuels-selon-martine-neddam-par-paule-mackrous, consulté le 9 janvier 2015.

Malraux, André, 1965, Le Musée imaginaire, Paris, Gallimard.

McLure, Helen, 2000, « The Wild Wild Web: the Mythic American West and the Electronic Frontier ", The Western Historical Quaterly, vol. 31, n 4, p. 457-476.

Miller, Paul D. (dir.), 2008, Sound Unbound. Sampling Digital Music and Culture, Cambridge (MA), MIT Press.

Navas, Eduardo, 2003, " Notes on Everything is a Remix ", Remixtheory.net, mis en ligne le 3 septembre 2003, http://remixtheory.net/?p=480, consulté le 9 janvier 2015.

Palmer, Amanda, 2013a, « Connecting the Dots, Dealing With Criticism and the Essence of Crowdfunding », [Blog], http://amandapalmer.net/blog/20130529/, consulté le 9 janvier 2015.

-, 2013b, « The Art of Asking », [Vidéo], TED, http://www.ted.com/talks/

amanda_palmer_the_art_of_asking.html, consulté le 9 janvier 2015.

Pettersen, Geneviève, 2013, « Il était une fois Madame Chose », Québec français, $n^{\circ}$ 68, p. 30-31.

Sherman, Aliza, 1998, A Woman's Guide to the Web. Cybergrrl!, New York, Ballantine.

Smithies, Lilly, 2013, « About », Creative Writing PhD, [Blog], http://

creativewriterphd.wordpress.com/about/, consulté le 9 janvier 2015.

Trunk, Penelope, 2007, « Use Blog as a Career Tool for Becomming Super Connected », Penelope Trunk, [Blog], http://blog.penelopetrunk.com/2007/06/24/blogging-supercharges-your-careerby-making-you-more-connected/, consulté le 9 janvier 2015. 
-, 2009, « How to decide How Much to Reveal About Yourself », Penelope Trunk [Blog], http:// blog.penelopetrunk.com/2009/07/21/how-to-decide-how-much-to-tell-about-yourself-on-yourblog/, consulté le 9 janvier 2015.

-, 2011, « The Psychology of Quitting », Penelope Trunk, [Blog], http://

blog.penelopetrunk.com/2011/12/28/the-psychology-of-quitting/, consulté le 9 janvier 2015.

Zambreno, Kate, 2007, Heroines, Cambridge (MA), MIT Press.

-, 2013, « 12-step Program for Author Ego », [Blog], http://

francesfarmerismysister.blogspot.ca/2013/05/12-step-program-for-author-ego.html (le blog est devenu privé après la rédaction de cet article).

Welby, Victoria, « bio », [section de site Web], http://victoriawelby.ca/bio, consulté le 9 janvier 2015.

-, « \#endsh », [Blog], http://victoriawelby.ca/les_identitaires/endsh, consulté le 9 janvier 2015.

\section{NOTES}

1. Les traductions comprises dans cet article sont les nôtres.

\section{RÉSUMÉS}

L'expression « écriveuses du Web » désigne ces femmes qui s'approprient avec courage et audace les espaces en ligne pour faire $d u$ Web leur territoire. Nous avons identifié deux notions permettant de problématiser leur pratique : l'abondance et le remix. Écrire sur le Web, c'est participer à l'abondance numérique, c'est-à-dire s'inscrire dans l'afflux de productions en ligne. Pour cela, les écriveuses trouvent des stratégies pour se connecter aux autres. Elles écrivent également pour garder le flux de leur créativité actif afin que leurs idées soient abondantes. Elles utilisent les écritures médiatiques qui, parfois, engendrent des remix. Ces derniers sont générés à partir d'une ou plusieurs autres productions culturelles et peuvent aussi s'appliquer à l'identité des écriveuses, qu'elles remixent afin d'explorer des formes d'écriture singulières.

Writers of the Web refer to these women appropriating online spaces to enter the Web and then write about it. Two concepts enable the analysis of their practice: the abundance and the remix. Writing on the Web is primarily being involved in digital abundance, that is, to participate in the influx of online productions. Writers also put themselves in a state of abundance: writing to keep a creative flow and share the fruits of their mental activity with others. This type of writing often takes the form of a remix, a collage created from one or several cultural productions. It can also take the form of an identity remix, that is, a way to explore an aspect of their personality and to test new forms of writing. 
INDEX

Mots-clés : écritures médiatiques, remix, flux, abondance, cowgirls, écriveuses

Keywords : Web media writing, abundance, writers

\section{AUTEUR}

PAULE MACKROUS

Université du Québec à Montréal 